Rich, P.M., L.H.Z. Weintraub, M.E. Ewers, T.L. Riggs, and C.J. Wilson. 2005. Decision support for water planning: the ZeroNet water-energy initiative. Proceedings of the American Society of Civil Engineers - Environmental \& Water Resources Institute (ASCE-EWRI) "World Water and Environmental Resources Congress 2005: Impacts of Global Climate Change", May 15-19, Anchorage, AK. LA-UR-05-1068.

\title{
Decision Support for Water Planning: the ZeroNet Water-Energy Initiative
}

\author{
Paul M. Rich ${ }^{1}$, Laura H.Z. Weintraub ${ }^{2}$, Mary E. Ewers ${ }^{3}$, \\ Thomas L. Riggs ${ }^{4}$, and Cathy J. Wilson ${ }^{5}$
}

${ }^{1}$ Earth and Environmental Science Division, Los Alamos National Laboratory, Los Alamos, NM 87545; PH (505)667-1850; FAX (505)667-1628; e-mail: pmr@lanl.gov

${ }^{2}$ Systech Engineering, Inc., 3180 Crow Canyon Pl, Suite 260, San Ramon, CA 94583; PH (925)355-1780; FAX (925)355-1778; e-mail: laura@systechengineering.com

${ }^{3}$ Department of Economics, University of New Mexico, Albuquerque NM 871310001; PH (505)667-6551; FAX (505)667-1628; e-mail: mewers@unm.edu

${ }^{4}$ Earth and Environmental Science Division, Los Alamos National Lab, Los Alamos, NM 87545; PH (505)667-1735; FAX (505)667-1628; e-mail: tlriggs@lanl.gov

${ }^{5}$ Earth and Environmental Science Division, Los Alamos National Laboratory, Los Alamos, NM 87545; PH (505)667-0202; FAX (505)665-3866; e-mail: cjw@lanl.gov

\section{Abstract}

Rapid population growth and severe drought are impacting water availability for all sectors (agriculture, energy, municipal, industry...), particularly in arid regions. New generation decision support tools, incorporating recent advances in informatics and geographic information systems (GIS), are essential for responsible water planning at the basin scale. The ZeroNet water-energy initiative is developing a decision support system (DSS) for the San Juan River Basin, with a focus on drought planning and economic analysis. The ZeroNet DSS provides a computing environment (cyberinfrastructure) with three major components: Watershed Tools, a Quick Scenario Tool, and a Knowledge Base. The Watershed Tools, based in the Watershed Analysis Risk Management Framework (WARMF), provides capabilities 1) to model surface flows, both the natural and controlled, as well as water withdrawals, via an engineering module, and 2) to analyze and visualize results via a stakeholder module. A new ZeroNet module for WARMF enables iterative modeling and production of "what if" scenario libraries to examine consequences of changes in climate, landuse, and water allocation. The Quick Scenario Tool uses system dynamics modeling for rapid analysis and visualization for a variety of uses, including drought planning, economic analysis, evaluation of management alternatives, and risk assessment. The Knowledge Base serves simultaneously as the "faithful scribe" to organize and archive data in easily accessible digital libraries, and as the "universal translator" to share data from diverse sources and for diverse uses. All of the decision tools depend upon GIS capabilities for data/model integration, map-based analysis, and advanced visualization. The ZeroNet DSS offers stakeholders an effective means to address complex water problems. 


\section{Rich et al.: Decision Support for Water Planning}

\section{What is the ZeroNet Water-Energy Initiative?}

The ZeroNet Water-Energy Initiative (http://zeronet.lanl.gov) addresses critical scientific challenges in understanding and planning for future water needs, with an initial focus in the San Juan Basin. The need for responsible water planning has been highlighted by the recent severe drought, during which freshwater surface flows have reached historical lows, and by concerns that such climatic extremes may be increasing in frequency and intensity due to global climate change (IPCC 2001). Herein we examine the ZeroNet Decision Support System (DSS), which includes three main components: an integrated set of watershed tools, a quick scenario tool, and a knowledge base. A DSS comprises much needed cyberinfrastructure, the computing environment for access to water management information, models, problem solving capabilities, and communication (Denshaw 1991, Heywood 1998, Goodchild and Rich 2005). We describe the capabilities of the ZeroNet DSS and provide initial results that demonstrate the utility for modeling freshwater surface flows and evaluating alternative management scenarios.

The ZeroNet Water-Energy Initiative seeks to meet new power generation needs with zero net new freshwater withdrawals by 2010. The ZeroNet initiative has three primary goals:

- reduce overall freshwater use in power generation cooling processes;

- augment freshwater with degraded and saline water for power generation cooling;

- use the best available data, models, and analysis tools to plan for responsible water management.

The ZeroNet partnership was initiated by Los Alamos National Laboratory (LANL), the Electric Power Research Institute (EPRI), and the Public Service Company of New Mexico (PNM), New Mexico's largest electricity and natural gas provider. The public-private partnership provides a unique opportunity to develop and test science and technology innovations at power generation facilities. Recently, the partnership has grown to include the University of New Mexico (UNM), Sandia National Laboratories (SNL), the Department of Energy National Energy Technology Laboratory (DOE NETL), and we are working to expand the initiative to include a broader range of industry and science partners.

ZeroNet stakeholders are diverse, and include local, state, and federal government agencies, industry, Native American tribes, irrigation districts, and private individuals. Stakeholders can use the ZeroNet DSS to understand the functioning of the San Juan River Basin, how new technologies and use of degraded water for power plant cooling affects freshwater availability, and how to formulate and evaluate management options for water shortage sharing schemes under drought conditions. The scientific information about various management options are presented in a logical and easy to understand manner so that the stakeholders can make informed decisions. 


\section{Rich et al.: Decision Support for Water Planning}

\section{What is the ZeroNet Decision Support System (DSS)?}

ZeroNet DSS is designed to provide tools that synthesize critical water supply and demand information and assist water owners and managers with planning for shortages. The ZeroNet DSS can answer critical management questions in a scientific and economic context. For example: Can new technology help to avert impending water shortages? How will changing climate (drought, warmer temperatures...) affect freshwater supply? How will watershed modification (vegetation, land use, fire...) affect freshwater supply? Can coordination and cooperation among water users (shortage sharing, conservation, water markets...) avert critical freshwater shortages?

The ZeroNet DSS has three major components: Watershed Tools, a Quick Scenario Tool (QST), and a Knowledge Base (Figure 1), based on foundations of stakeholder input, watershed data, and geographic information system (GIS) capabilities. All of the DSS tools are being made available without charge to stakeholders as stand-alone programs that run on personal computers under Microsoft Windows ${ }^{\mathrm{TM}}$. The DSS user interfaces are designed for ease of use, and users can conveniently examine underlying assumptions, data, and their own scenarios.

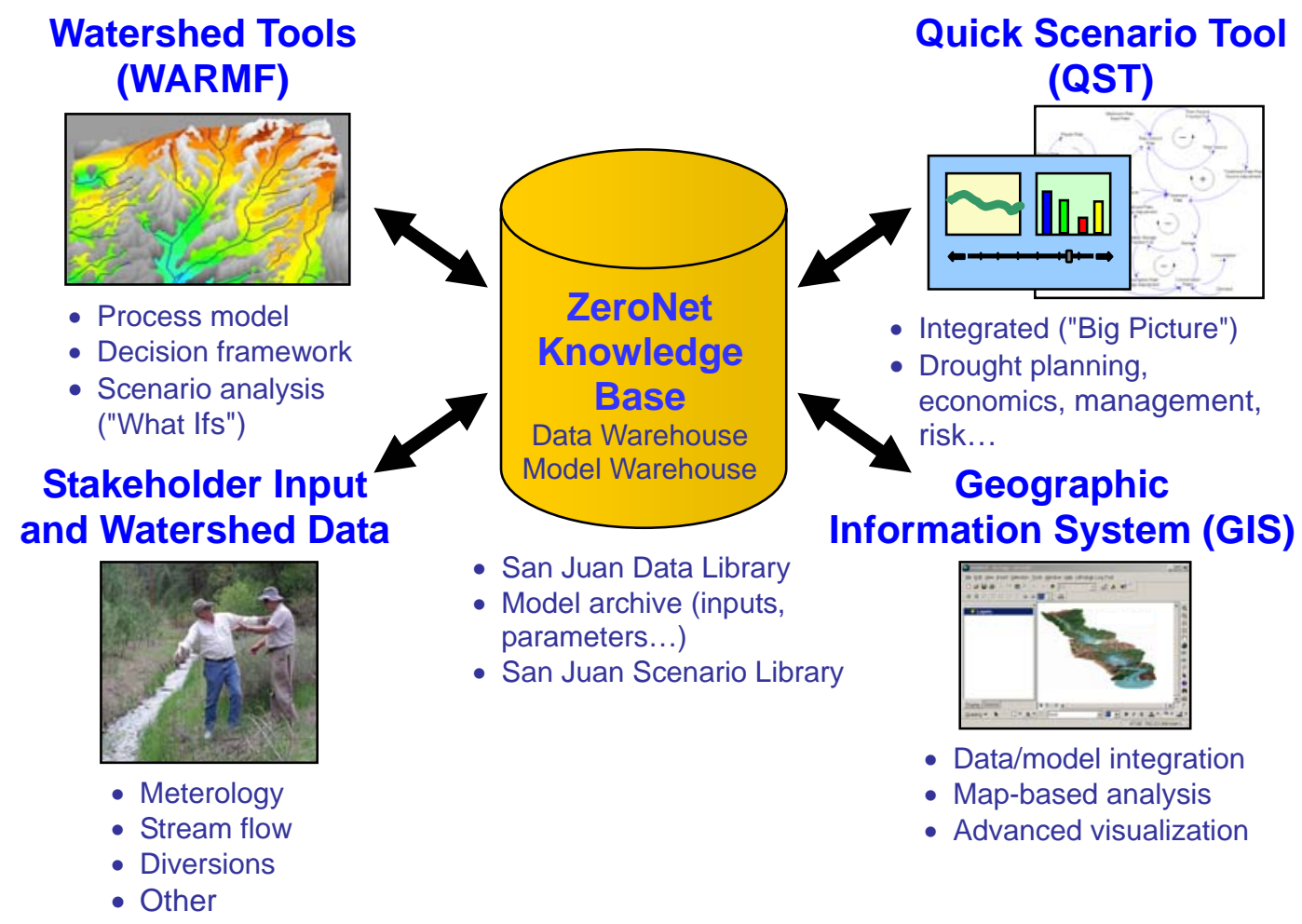

Figure 1. The ZeroNet Decision Support System (DSS). 


\section{Rich et al.: Decision Support for Water Planning}

\subsection{Watershed Tools (WARMF)}

The Watershed Analysis Risk Management Framework (WARMF) watershed tools calculate surface and subsurface flows, as well as water quality (Chen et al. 2001, Keller 2000, 2001, Weintraub et. al. 2001) (Figure 2). WARMF has been applied to over fifteen watersheds in the United States and internationally. In addition to simulating flow, WARMF simulates water quality constituents including temperature, total suspended solids, coliform bacteria, biochemical oxygen demand (BOD), dissolved oxygen (DO), nutrients (phosphorus and nitrogen), chlorophyll, and others. WARMF was originally designed to support modeling and planning for total maximum daily loads (TMDLs), the amount of a particular pollutant that a particular stream, lake, estuary or other water body can handle without violating state water quality standards.
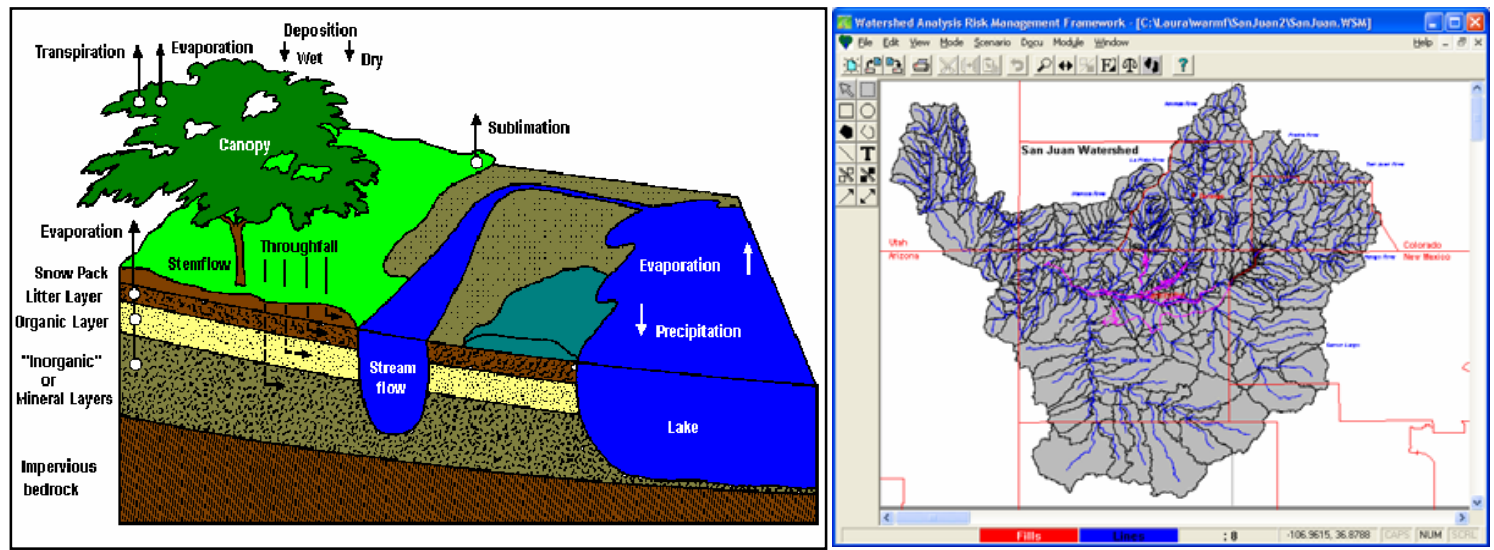

Figure 2. The WARMF watershed tools include process models to simulate freshwater flow (left) and access via a graphical user interface (right).

Design: WARMF currently consists of five integrated modules (Engineering, Consensus, TMDL, Data, and Knowledge). WARMF has two main components, the simulator which is written in FORTRAN and the graphical user interface (GUI) which is written in $\mathrm{C}++$. The GUI provides a mechanism to view input and output in dialogs, tables, and graphs. The simulator contains all of the algorithms and calculations used to run model simulations. Enhancements of WARMF in support of the ZeroNet initiative include iterative modeling and production of "what if" scenario libraries to examine consequences of changes in climate, landuse, and water allocation. This is being accomplished by development of a ZeroNet module, a Batch Scenario Tool, and improvements to the Engineering and Knowledge modules. Although the Consensus and Data modules of WARMF will be used during the WARMF application to the San Juan basin, no modifications to these modules are planned.

Engineering Module: At the heart of WARMF is the Engineering module that contains a dynamic watershed simulation model that calculates daily surface runoff, ground water flow, non-point source loads, hydrology, and water quality of river segments and stratified reservoirs. In WARMF, a watershed is divided into a 


\section{Rich et al.: Decision Support for Water Planning}

network of land catchments, river segments, and reservoir layers. Land catchments are further divided into land surface and soil layers. These watershed compartments are seamlessly connected for hydrologic and water quality simulations. The land surface is characterized by its land uses and cover, which may include forested areas, agricultural lands, or urbanized cities. Daily precipitation, which includes rain and snow, is deposited on the land catchments. WARMF performs daily simulations of snow and soil hydrology to calculate surface runoff and groundwater accretion to river segments. The water is then routed from one river segment to the next downstream river segment until it reaches the watershed terminus. Associated point and nonpoint loads are also routed through the system. Heat budget and mass balance calculations are performed to calculate the temperature and concentrations of various water quality constituents in each soil layer, river segment, and lake layer.

ZeroNet Module: The new ZeroNet module for WARMF (Figure 3) is being developed to provide stakeholder access to set up simulations, view results, and export results to the ZeroNet Knowledge Base. The module guides users through a series of six steps to run simulations and view model output. First, the user sets up the scenario to simulate. Users can either run a single scenario or a batch of scenarios. Second, the user specifies the goals of an alternative, both as a "designated use" (e.g., fish habitat) and as "criteria" meet (e.g., minimum flow), with provision to interactively choose where this applies via a GIS map. Third, the user specifies management modifications to flows in a spreadsheet. Fourth, the user launches the simulation. Fifth, the user views the results in terms of hydrographs and GIS maps (e.g. green for meeting flow targets, red when violating targets). And sixth, the user exports the results.

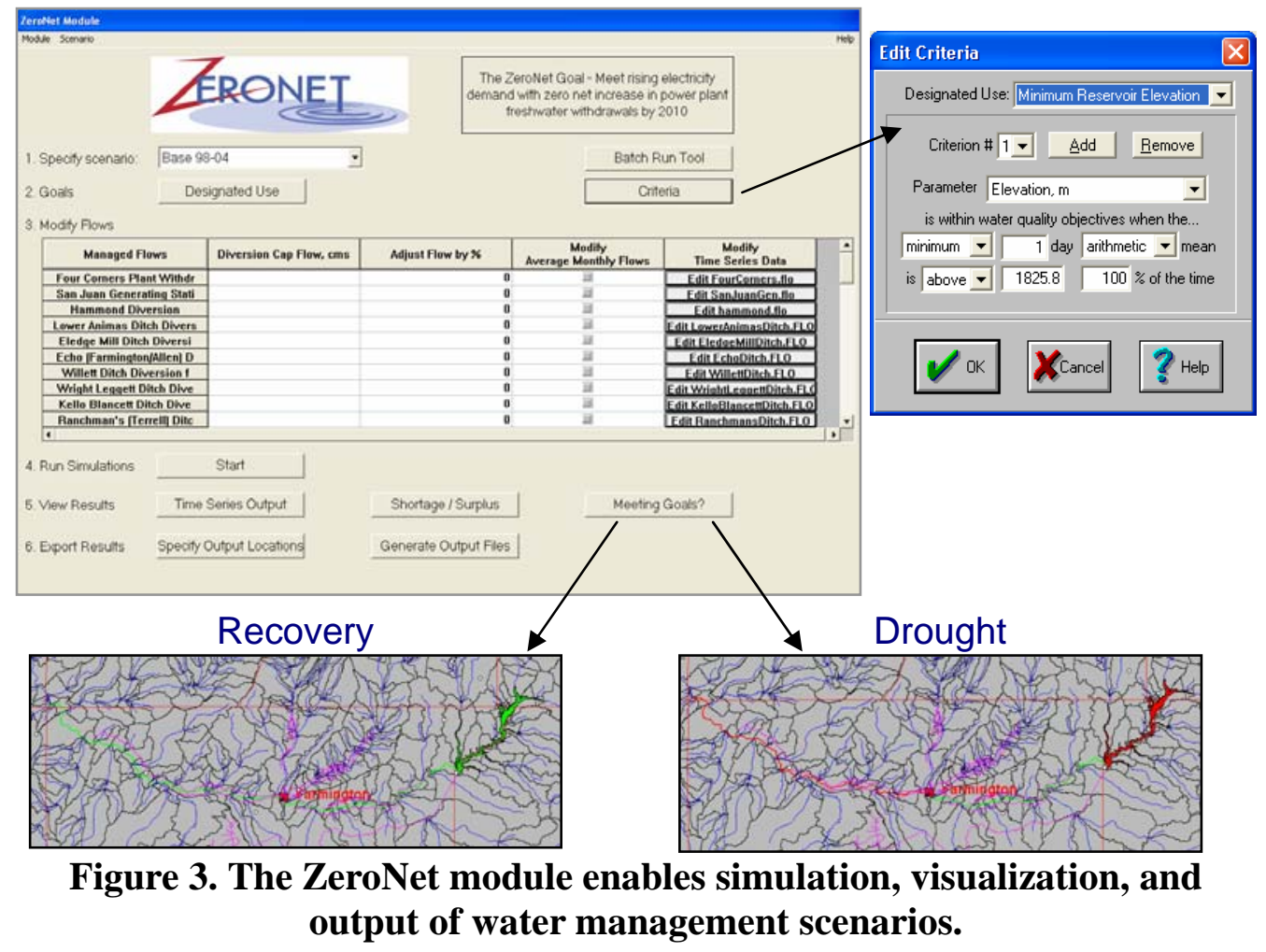




\section{Rich et al.: Decision Support for Water Planning}

Batch Scenario Tool: The Batch Scenario Tool is being developed to enable a user to run multiple simulations in a single step (Table 1). The purpose of running multiple scenarios is to build scenario libraries for use via the QST. The QST uses WARMF output to examine the responses to factors such as meteorology, managed flow, and point source control.

Table 1. Example parameters for batch scenarios.

\begin{tabular}{|c|c|c|c|}
\hline Parameter & Low & Medium & High \\
\hline Meteorology-precip & $-15 \%$ & Historical & $+15 \%$ \\
\hline Meteorology-temp & $-15 \%$ & Historical & $+15 \%$ \\
\hline
\end{tabular}

\subsection{ZeroNet Quick Scenario Tool (QST)}

The QST provides convenient means to ask "what if" questions, and thus represents an important tool for learning about watershed processes and making water management decisions (Figure 4). Initial implementation of the QST focuses on the ability to analyze scenarios based on assumptions concerning climate and water supply, reservoir balance, and surface water diversion. The QST is based in the VenSim $^{\mathrm{TM}}$ system dynamics software, which enables higher level integration of models, and linking of processes based on stocks, flows, and feedbacks (positive and negative) that reproduces, as accurately as possible, the interactions between the natural hydrologic system and human economic demands on the hydrologic system. A hallmark of the QST is the ability to dynamically vary values of a single input variable (such as precipitation, or amount of water diverted) using "sliders", and to visualize the consequences in terms such as water supply or cost.
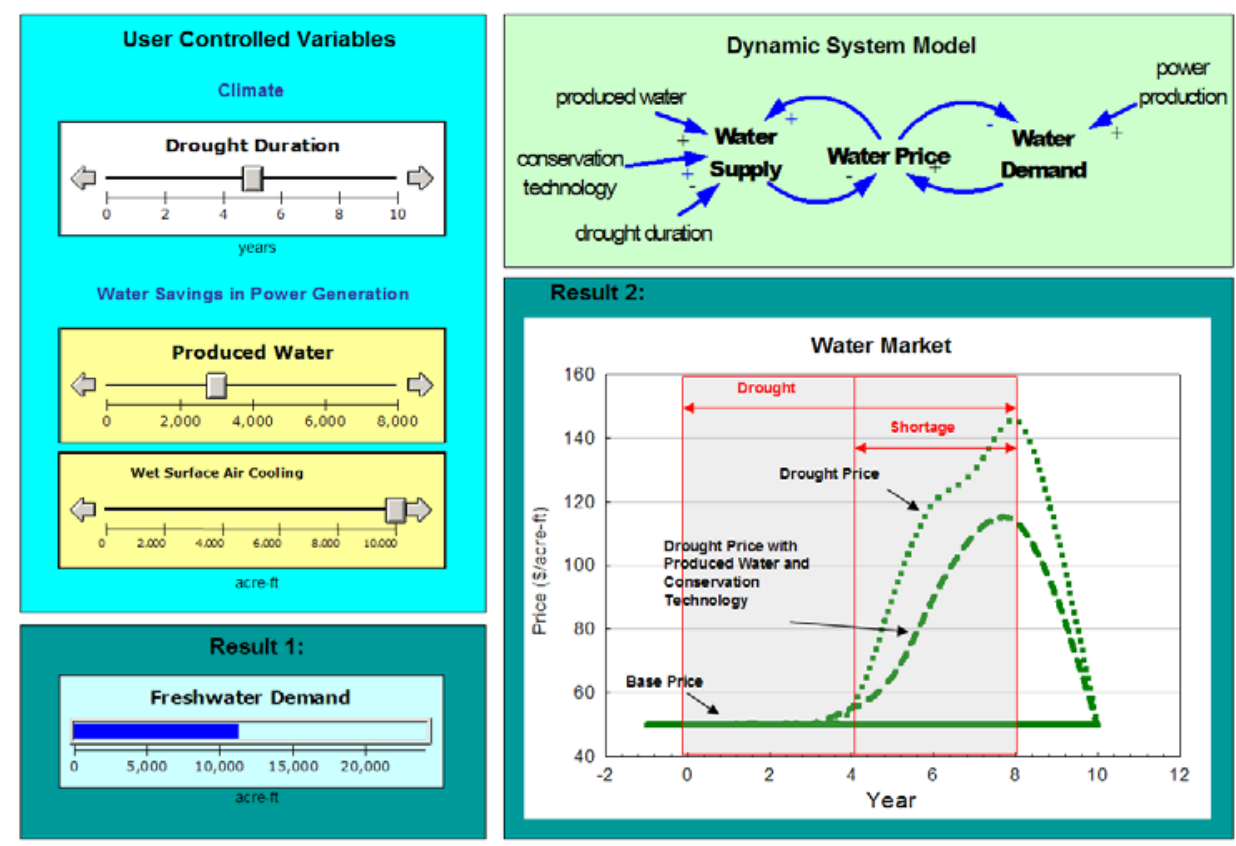

Figure 4. The ZeroNet Quick Scenario Tool enables rapid analysis and visualization. In this case the user can adjust climate and water savings sliders to explore influences of water demand and price. 


\section{Rich et al.: Decision Support for Water Planning}

QST Capabilities: The initial prototype QST is being developed to enable examination of either historical or hypothetical scenarios based on five sets of user controlled input variables for the San Juan basin:

- Water supply and climate: to explore influences of natural surface flow levels and climate variables (precipitation and temperature);

- Reservoir balance: to explore water storage in the Navajo Reservoir, based on inflows, diversions, and release rates;

- Diversion levels: to explore influences of diversions on supply.

- Energy water use: to explore economic impacts on energy production (typically cost of water or revenues).

- Municipal water use: to explore economic impacts on municipalities, based on management practices and population growth.

- Agricultural water use: to explore economic impacts on agriculture, measured as the value of lost crop production.

Scenario Libraries: Often it is not practical to perform simulations in real time. By pre-calculating series of scenarios and storing them in scenarios libraries, it becomes practical to examine a broad range of scenarios in near real time. Within limits, additional scenarios can be calculated from the scenario by intelligent interpolation and extrapolation.

\subsection{ZeroNet Knowledge Base}

The ZeroNet Knowledge Base is the "centerpiece" of the ZeroNet DSS (Figure 5).

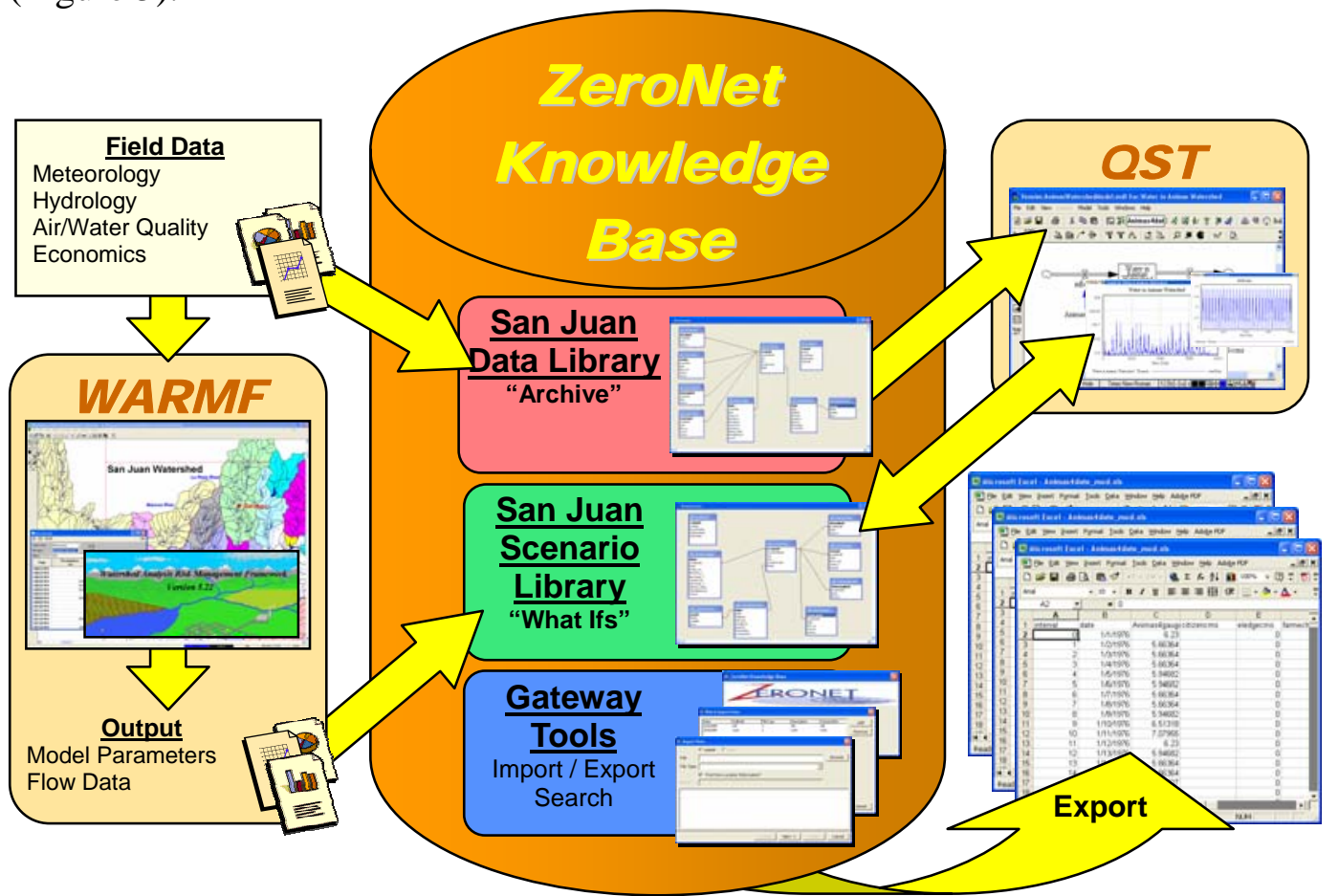

Figure 5. The ZeroNet Knowledge Base contains digital libraries of data and model scenarios, with gateway tools for import, export, and search. 


\section{Rich et al.: Decision Support for Water Planning}

The Knowledge Base serves simultaneously as the "faithful scribe" to organize and archive, as the "universal translator" to share data from diverse sources and for diverse uses, and as the "friendly traffic controller" to direct data flow and ensure access. The Knowledge Base has three main components: a data warehouse, a model warehouse, and gateway tools.

Data-Model Integration: An important function of the ZeroNet Knowledge Base is integration of diverse data, models, and model output from diverse sources. The Knowledge Base provides "loose coupling", in that it provides the means for data sharing among widely distributed data sources, diverse models (WARMF, QST...), and applications with different data formats.

Data Warehouse: A data warehouse archives, manages, and provides access to data (Keating et al. 2003, Witkowski et al. 2003, 2005). In the case of ZeroNet, the model warehouse comprises databases that constitute the San Juan Data Library. These data include meteorological records (precipitation, temperature...), hydrological records (flows, reservoir levels...), diversions (actual, rights...), and economics (technology costs, water market values...). Because these data are scattered and often difficult to find, compilation of key data in one location represents a resource of value to a broad set of stakeholders. At present the data reside in a variety of different formats, including WARMF-specific data files and Excel spreadsheets. We are working to migrate key data into Microsoft Access ${ }^{\mathrm{TM}}$ tables, and eventually plan to migrate the San Juan data library to an enterprise Oracle ${ }^{\mathrm{TM}}$ database.

Model Warehouse: The concept of a model warehouse is similar to that of a data warehouse, but pertains to models, model parameters, and scenario libraries. Management of the model warehouse requires standardized documentation of models and model outputs (model metadata). The San Juan Scenario Library will store simulations concerning climate (drought, increased temperature...), watershed modification (fire...), coordination/cooperation (shortage sharing, conservation...), economics (projected costs, market behavior...), and resulting water supply (flows, reservoir levels, shortages...).

Gateway Tools: Gateway tools perform three roles for use of the ZeroNet Knowledge Base: import, export, and search. Our effort is currently focused on import from the San Juan data library to WARMF and the QST, export from WARMF and the QST, and search using queries on metadata.

\section{Initial Results}

An initial step in calibration and validation of the hydrological models for the San Juan Basin involves matching observed values and WARMF simulations in hydrographs that depict flow rates through time (Figure 6). WARMF is able to match historical values with a high degree of reliability under diverse climatic conditions. 
Rich et al.: Decision Support for Water Planning

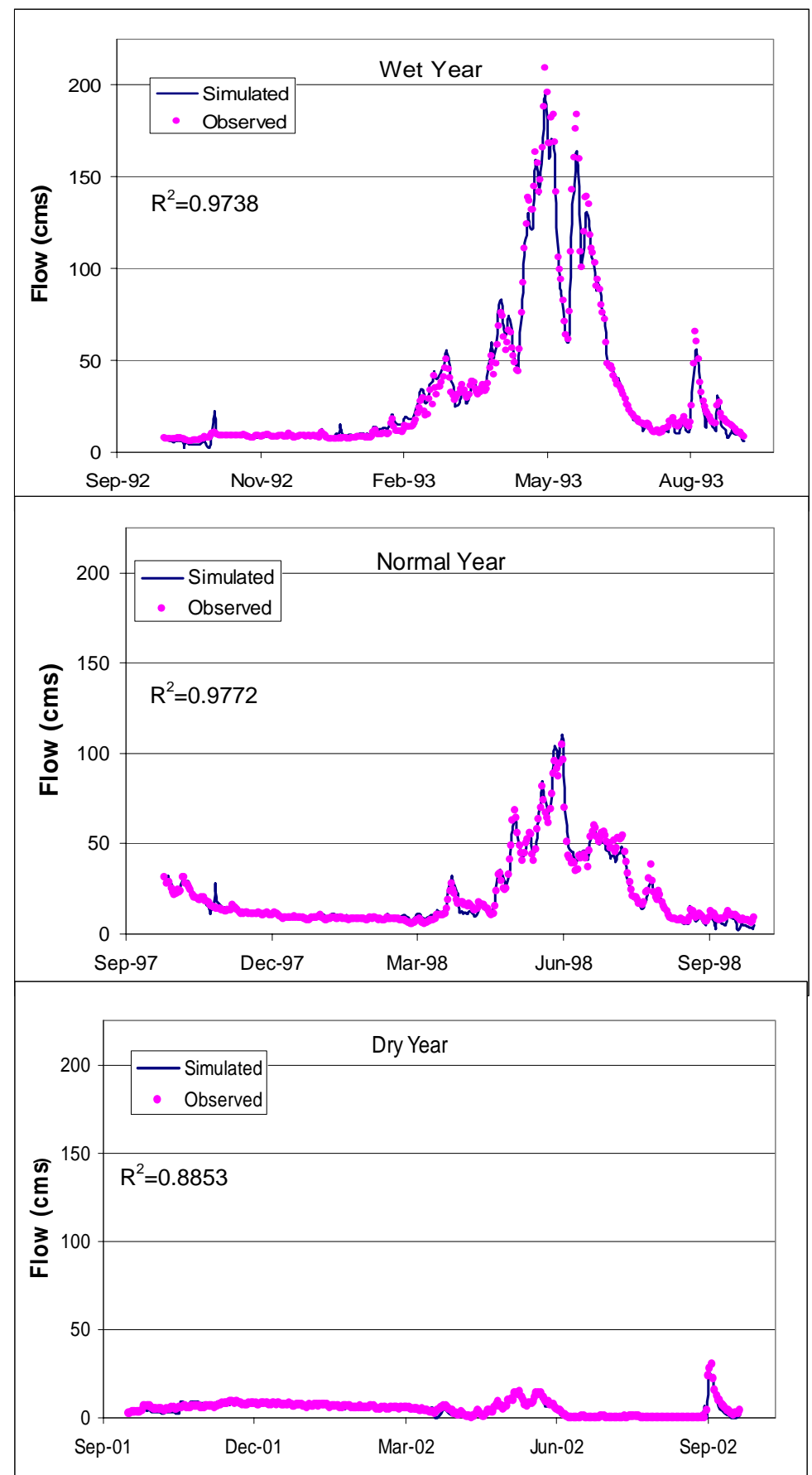

Figure 6. Hydrographs for wet, normal, and dry years of the Animas River.

Simulations were performed to project Navajo Reservoir levels for 2005-2009 under various meteorological and water management scenarios (Figure 7). If drought conditions continue at similar levels for the next five years, reservoir levels are expected to drop below the critical allowable level, even with levels of shortage sharing agreed to in 2003, or with this shortage sharing and 5000 acre-ft of water savings by using produced water from oil and gas operations. However, if the next 
five years have typical precipitation, then a recovery to near pre-drought levels is expected.

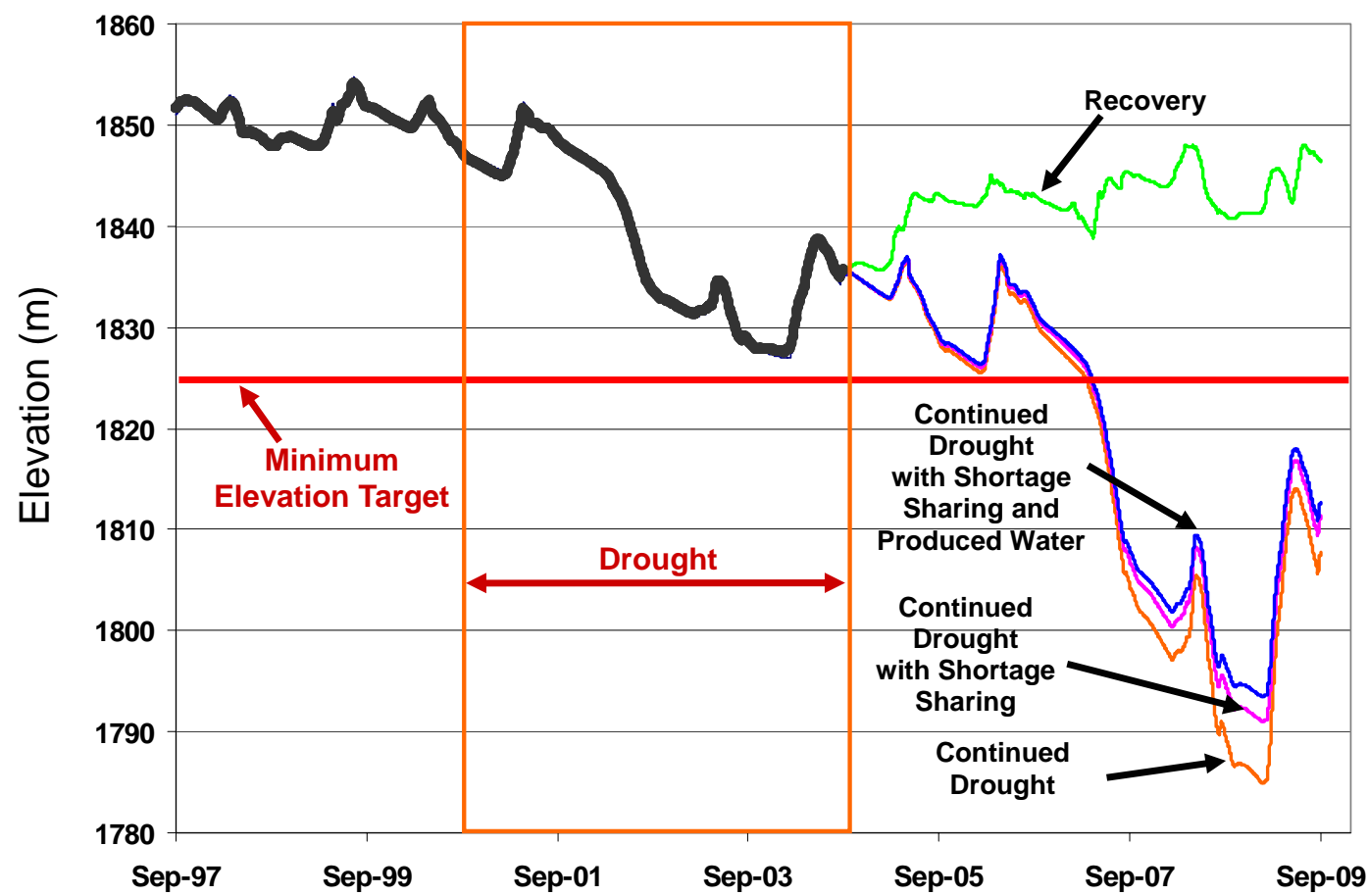

Figure 7. Navajo Reservoir levels based on historical data until present, and projected into the future under four scenarios: continued drought, continued drought with shortage sharing, continued drought with shortage sharing and produced water savings, and recovery.

\section{Conclusion}

The ZeroNet DSS, with foundations in GIS and stakeholder input, provides a general framework for decision support applied to basin level modeling and management. The design consists of three elements: a comprehensive set of watershed tools, a quick scenario tool, and integration via a basin-specific knowledge base. Strengths include sound physics-based models of hydrology, integration of diverse data and models, incorporation of economics, and user-friendly access on PC platforms. Further work, both empirical and theoretical, is needed to validate the models and to assess relative advantages of the use of scenario libraries versus real-time calculations. In the face of growing concern over climate change, limited water resources, and competing demands, such integrative DSS tools can enable better understanding of complex interconnected systems, and enable better decisions. 


\section{Rich et al.: Decision Support for Water Planning}

\section{Acknowledgements}

This work was supported by the Department of Energy National Energy Technology Laboratory (DOE NETL), the Los Alamos National Laboratory Earth and Environmental Science Division (LANL EES), the Public Service Company of New Mexico (PNM), and the Electric Power Research Institute (EPRI). Thanks to members of the San Juan Shortage Sharing group for input concerning stakeholder needs. Thanks to Shawn Williams for assistance in compiling San Juan basin data. Thanks to Joel Herr and Limin Chen for assistance with enhancements to WARMF. Thanks to Thomas Shumaker, Amy Miller, and Anthony Mancino for preparing the initial ZeroNet website and background brochures. And thanks to Marc Witkowski, Gordon Keating, and members of the ZeroNet team for valuable input on the ZeroNet DSS design, and for providing editorial comments to improve this publication.

\section{References}

Chen, C.W., J. Herr, and L. Weintraub. 2001. Watershed Analysis Risk Management Framework (WARMF): Update One - a decision support system for watershed analysis and total maximum daily load calculation, allocation and implementation. Publication No. 1005181, EPRI, Palo Alto, CA.

Densham, P.J. 1991. Spatial decision support systems. In: D.J. Maguire, M.F. Goodchild, and D.W. Rhind (eds.). Geographical information systems: principles and applications. John Wiley and Sons, New York: 403-412.

Heywood, I., S. Cornelius and S. Carver. 1998. An introduction to geographical information systems. Addison Wesley, Longman, NY, 297 pp.

Goodchild, M.F. and P.M. Rich. 2005. Space, time, and the emerging cyberinfrastructure. manuscript to be submitted for peer review.

Intergovernmental Panel on Climate Change (IGCC). 2001. Climate change 2001: synthesis report. R.T. Watson and the Core Writing team (eds). Cambridge University Press, New York.

Keating, G.N., P.M. Rich, and M.S. Witkowski. 2003. Challenges for Enterprise GIS. URISA Journal 15(2):25-39.

Keller, A. 2000. Peer review of WARMF - an evaluation of WARMF for TMDL applications by independent experts using USEPA guidelines. Technical Report 2000.1000252, EPRI, Palo Alto, CA.

Keller, A. 2001. Peer review of the acid mine drainage module of WARMF - an evaluation of WARMF/AMD using USEPA guidelines. Technical Report 2001.1005182, EPRI, Palo Alto, CA.

Weintraub, L.H.Z., C.W. Chen, J. Herr. 2001. Demonstration of WARMF: a decision support tool for TMDL Development. Proceedings of WEF TMDL Science Issues Conference, March 4-7, 2001. St. Louis, MO.

Witkowski, M.S., P.M. Rich, and G.N. Keating. 2003. A prototype for enterprise GIS. Los Alamos National Laboratory Report, LA-14027, Los Alamos, NM.

Witkowski, M.S., P.M. Rich, and G.N. Keating. 2005. Enterprise GIS: definition, requirements, and metrics of success. submitted for peer review. 